\title{
Prevention of Nosocomial Transmission of Norovirus by Strategic Infection Control Measures
}

\author{
Vincent C. C. Cheng, MD; $;^{1,2}$ Lisa M. W. Wong, MSc; Josepha W. M. Tai, MHSc; ${ }^{2}$ Jasper F. W. Chan, MD; ${ }^{1}$ \\ Kelvin K. W. To, MD; ${ }^{1}$ Iris W. S. Li, MD; ${ }^{1}$ Ivan F. N. Hung, MD; ${ }^{3}$ K. H. Chan, PhD; ${ }^{1}$ \\ P. L. Ho, MD; ${ }^{1}$ K. Y. Yuen, $\mathrm{MD}^{1}$
}

BACKGROUND. Nosocomial outbreaks of norovirus infection pose a great challenge to the infection control team.

METHODs. Between November 1, 2009, and February 28, 2010, strategic infection control measures were implemented in a hospital network. In addition to timely staff education and promotion of directly observed hand hygiene, reverse-transcription polymerase chain reaction for norovirus was performed as an added test by the microbiology laboratory for all fecal specimens irrespective of the request for testing. Laboratory-confirmed cases were followed up by the infection control team for timely intervention. The incidence of hospitalacquired norovirus infection per 1,000 potentially infectious patient-days was compared with the corresponding period in the preceding 12 months, and the incidence in the other 6 hospital networks in Hong Kong was chosen as the concurrent control. Phylogenetic analysis of norovirus isolates was performed.

RESUlts. Of the 988 patients who were tested, 242 (25\%) were positive for norovirus; 114 (47\%) of those 242 patients had norovirus detected by our added test. Compared with the corresponding period in the preceding 12 months, the incidence of hospital-acquired norovirus infection decreased from 131 to 16 cases per 1,000 potentially infectious patient-days $(P<.001)$, although the number of hospitalacquired infections was low in both the study period $(n=8)$ and the historical control periods $(n=11)$. The incidence of hospital-acquired norovirus infection in our hospital network ( 0.03 cases per 1,000 patient-days) was significantly lower than that of the concurrent control ( 0.06 cases per 1,000 patient-days) $(P=.015)$. Forty-three $(93 \%)$ of 46 norovirus isolates sequenced belonged to the genogroup II.4 variant.

CONCLUSions. Strategic infection control measures with an added test may be useful in controlling nosocomial transmission of norovirus.

Infect Control Hosp Epidemiol 2011;32(3):229-237

Norovirus is a nonenveloped, single-stranded RNA virus belonging to the family of Caliciviridae, which is a causative agent for acute gastroenteritis, often referred to as "winter vomiting disease." Norovirus can cause major outbreaks in both communities and hospitals, with a high clinical attack rate due to its low infectious dose of 10-100 particles, relative resistance to disinfecting agents, and environmental survival of up to 2 days. ${ }^{1}$ With the emergence of a specific genogroup II. 4 variant since 2002, there has been a dramatic increase in the number of reported outbreaks all over the world, ${ }^{2}$ including in Hong Kong. ${ }^{3}$ In the summer of 2006, there were 36 nosocomial outbreaks of norovirus in 19 of 41 public hospitals in Hong Kong affecting 218 patients, most of them elderly. ${ }^{4}$ Control of nosocomial outbreaks of norovirus infection often requires infection control measures that include closure of a ward, contact isolation precaution, and extensive environmental disinfection., ${ }^{5,6}$ These measures disrupt patient care and increase both the workload of frontline staff and the costs to healthcare institutions. ${ }^{7}$

During the pandemic outbreak of infection with the novel influenza A H1N1 virus, we successfully demonstrated the effectiveness of enhanced infection control measures in preventing nosocomial transmission of this novel virus. ${ }^{8}$ Here, we further tested strategic infection control measures targeting norovirus during the winter, when the average temperature was $16^{\circ} \mathrm{C}$ (range, $14^{\circ} \mathrm{C}-20^{\circ} \mathrm{C}$ ) and the relative humidity was around $70 \%{ }^{9}$ By incorporating an "added test" for norovirus in the microbiology laboratory, the sequence of infection control actions by the infection control team prevented any nosocomial outbreaks of norovirus infection in our hospital while the number of outbreaks of norovirus infection in the other public hospitals in Hong Kong remained unchanged this year.

Affiliations: 1. Department of Microbiology, Queen Mary Hospital, Hong Kong Special Administrative Region, China; 2. Infection Control Unit, Queen Mary Hospital, Hong Kong Special Administrative Region, China; 3. Department of Medicine, Queen Mary Hospital, Hong Kong Special Administrative Region, China.

Received June 2, 2010; accepted August 18, 2010; electronically published February 15, 2011.

(C) 2011 by The Society for Healthcare Epidemiology of America. All rights reserved. 0899-823X/2011/3203-0005\$15.00. DOI: 10.1086/658330 


\section{METHODS}

\section{Infection Control Measures for Norovirus}

In collaboration with the stakeholders, including hospital administrators, clinicians, and nursing colleagues, strategic infection control measures were established to prevent nosocomial transmission of norovirus infection. Open staff forums and special education sessions were arranged by the hospital administration and infection control team. Fecal specimens were collected for all hospitalized patients who had diarrheal symptoms on admission or during hospitalization. Reversetranscription polymerase chain reaction (RT-PCR) for the ORF1 gene of norovirus was performed upon request by the attending clinician (the "ordered test"). However, RT-PCR for norovirus was also performed as an added test by the microbiology laboratory for all fecal specimens that were requested for bacterial culture, Clostridium difficile culture, or cytotoxin, and rotavirus antigen detection without a request for norovirus detection. The infection control team checked with the microbiology laboratory on a daily basis for any test results positive for norovirus.

Cases were assessed at the bedside to confirm that patients had symptomatic diarrhea when fecal specimens were collected and to determine the route of acquisition. Communityacquired infection was defined as present if a patient had diarrheal symptoms upon or within 48 hours after admission and had no history of contact with any confirmed case in the hospital. Hospital-acquired infection was defined as present if a patient had onset of diarrhea after 48 hours of hospitalization.

The source of nosocomial transmission of norovirus was investigated by the infection control team with the aim to identify areas for improvement. The infection control team also advised healthcare workers on isolation and contact precautions for all laboratory-confirmed cases. An infection control action cascade was introduced in our hospital. Briefly, infection control nurses made daily, unannounced visits to areas at high risk for outbreaks of norovirus infection to detect any lapse in infection control practice. An infection control nurse contacted the high-risk areas by phone during public holidays and weekends to ensure that appropriate patient placement was made for all patients with nosocomial onset of diarrhea. A high-risk area was defined as any clinical unit or ward in which nosocomial transmission of diarrheal pathogens had been reported in the past 12 months. ${ }^{10} \mathrm{~A}$ nosocomial outbreak was defined as 3 or more laboratoryconfirmed cases of hospital-acquired norovirus infection in the same ward. When nosocomial outbreaks of norovirus infection occurred in the other hospitals in Hong Kong, our infection control team would immediately review relevant patient care practices and hospital environments to lower the corresponding risk factors, if present, that may contribute to a nosocomial outbreak.

Hand hygiene using an alcohol-based hand rub (World
Health Organization formulation) was promoted in 2006 and fully implemented in our hospital in 2007. Directly observed hand hygiene practice was promoted, especially in the highrisk areas as previously described. ${ }^{11}$ Compliance with hand hygiene practices has been regularly audited by the infection control team according to a predetermined protocol. ${ }^{12}$ The consumption data of alcohol-based hand rub retrieved from the pharmacy was calculated in terms of liters per 1,000 patient-days.

\section{Epidemiology of Norovirus in Queen Mary Hospital}

This study was performed in Queen Mary Hospital, a 1,400bed tertiary referral university-affiliated hospital with 3 adult isolation wards and 1 pediatric isolation ward, under the governance of hospital network A in Hong Kong. Except for general pediatric patients who were routinely admitted into isolation facilities when they presented with diarrheal illness, all other patients, including surgical pediatric patients, were placed in the nonisolation facilities upon admission in view of the limitation of isolation facilities. Hospitalized patients were transferred to 4 chronic care hospitals designated for rehabilitation and extended medical care with a total of 1,200 beds within hospital network A when their acute conditions stabilized. Hospital network A is estimated to provide clinical service to a population of approximately 0.53 million. Our microbiology laboratory provides service to 5 hospitals in hospital network A. From November 1, 2009, to February 28, 2010, all fecal specimens sent to the microbiology laboratory were subjected to norovirus RT-PCR as either an ordered test or an added test, with a turnaround time of 1 day. The overall positive rate of norovirus infection in hospital network A was described. The epidemiology and nosocomial transmission of norovirus infection diagnosed in Queen Mary Hospital was analyzed. The number of potentially infectious patient-days for norovirus was defined as the interval from onset of diarrhea upon admission or during hospitalization to the implementation of infection control practice, including cohort nursing or single room isolation. The number of hospital-acquired norovirus infections in the corresponding period (November-February) in the preceding 12 months was retrieved for comparison to assess the effectiveness of our infection control measures.

\section{Nosocomial Outbreak of Norovirus Infection in Hong Kong}

In Hong Kong, there are 7 hospital networks comprising 41 public hospitals under the management of the Hospital Authority, which serves over $90 \%$ of the 7 million people in Hong Kong. The infection control officer of a hospital is responsible for monitoring the occurrence of nosocomial outbreaks and reporting to the Hospital Authority if the outbreak might have a public health impact. The number of exposed patients and staff in each outbreak of norovirus infection was retrieved from the Hospital Authority. The number and clin- 
ical attack rates of the nosocomial outbreaks in all 7 hospital networks in the corresponding period of 2009 were reported. The incidence of hospital-acquired norovirus infection in the hospital network was expressed in 1,000 patient-days.

Molecular Diagnosis and Phylogenetic Analysis of Norovirus by Real-Time RT-PCR

A $10 \%(\mathrm{wt} / \mathrm{vol})$ fecal suspension was prepared with viral transport medium and clarified by centrifugation at $3,000 \mathrm{~g}$ for 20 minutes. Total nucleic acid was extracted from $250 \mu \mathrm{L}$ of the $10 \%$ clarified fecal suspension with NucleiSens easyMAG instrument (bioMerieux, Boxtel, Netherlands) according to the manufacturer's instructions. Extracted RNA was reverse-transcribed to complementary DNA (cDNA) using the SuperScript II kit (Invitrogen, San Diego, CA) with random primer as previously described. ${ }^{13}$ Real-time RT-PCR for norovirus was performed by using primers to amplify the ORF1-ORF2 junction region and probes for genotype 1 and II as previously described with modification. ${ }^{14}$ PCR amplification was performed with a LightCycler 2.0 (Roche Diagnostic) under the following conditions: initial denaturation at $95^{\circ} \mathrm{C}$ for 10 minutes (preamplification hot start), and then 50 cycles of amplification with denaturation at $95^{\circ} \mathrm{C}$ for 10 seconds, annealing at $56^{\circ} \mathrm{C}$ for 15 seconds, and extension at $72^{\circ} \mathrm{C}$ for 12 seconds. Amplification data were collected and analyzed with LightCycler 4.1 software (Roche Diagnostic).
Published sequences of representative norovirus strains from GenBank were included for alignments and phylogenetic analysis. The accession numbers are New Orleans 1905/ 2009/USA (GU445325), NSW001P/2008/AU (GQ845367), DOUG4770/GII.4 (AF406793), Burwash Landing/331/1995/ USA (AF414425), FUMI/2010/JP (AB543808), Hu/GII/281/ 2006/HKG (EF121839), Hu/GII/733/2006/HKG (EF121840), Lordsdale/1993/UK (X86557), Camberwell/1994/AU (HCU46500), Miami/292/1994/US (AF414410), Gwynedd/ 273/1994/US (AF414409), Toronto/24/1999/CA (U02030), Mexico/1989/MX/ (U22498), Lionville/247/1993/US (AF414411), Richmond/283/1994/US (AF414419), White_river (AF414423), Melksham/1989/UK (X81879), Hu/ GII/NoV750/2004/CA (EF078288), Saitama T80GII/02/JP (AB112293), Saitama U25 (AB039780), Hu/GII.4/Nijmegen115/2006 (EF126966), Hu/GII.4/DenHaag89/2006 (EF126965)， Cairo2/2006 (EU876882), Cairo4/2006 (EU876884), Ichikawa/2005 (AB294787), Sakai/04-179/2005 (AB220922), Kashiwa/060802/2006 (AB2947789), Kashiwa/ 060802/2006 (EF126964), Aomori1/2006 (AB447432), Almelo039/2004 (AB303938), Hu/GII.4/2004 (AY883096), Apeldoorn023/2003 (AB303931), Kaiso/030556/2003 (AB294779/2001), Houston/TCH186/2002 (EU310927), Stockholm/19865/2008 (AB492092), Apeldoorn317/2007 (AB445395), Dijon/E3743/2009 (GQ246798), Dijon/E4032/ 2009 (GQ246801).
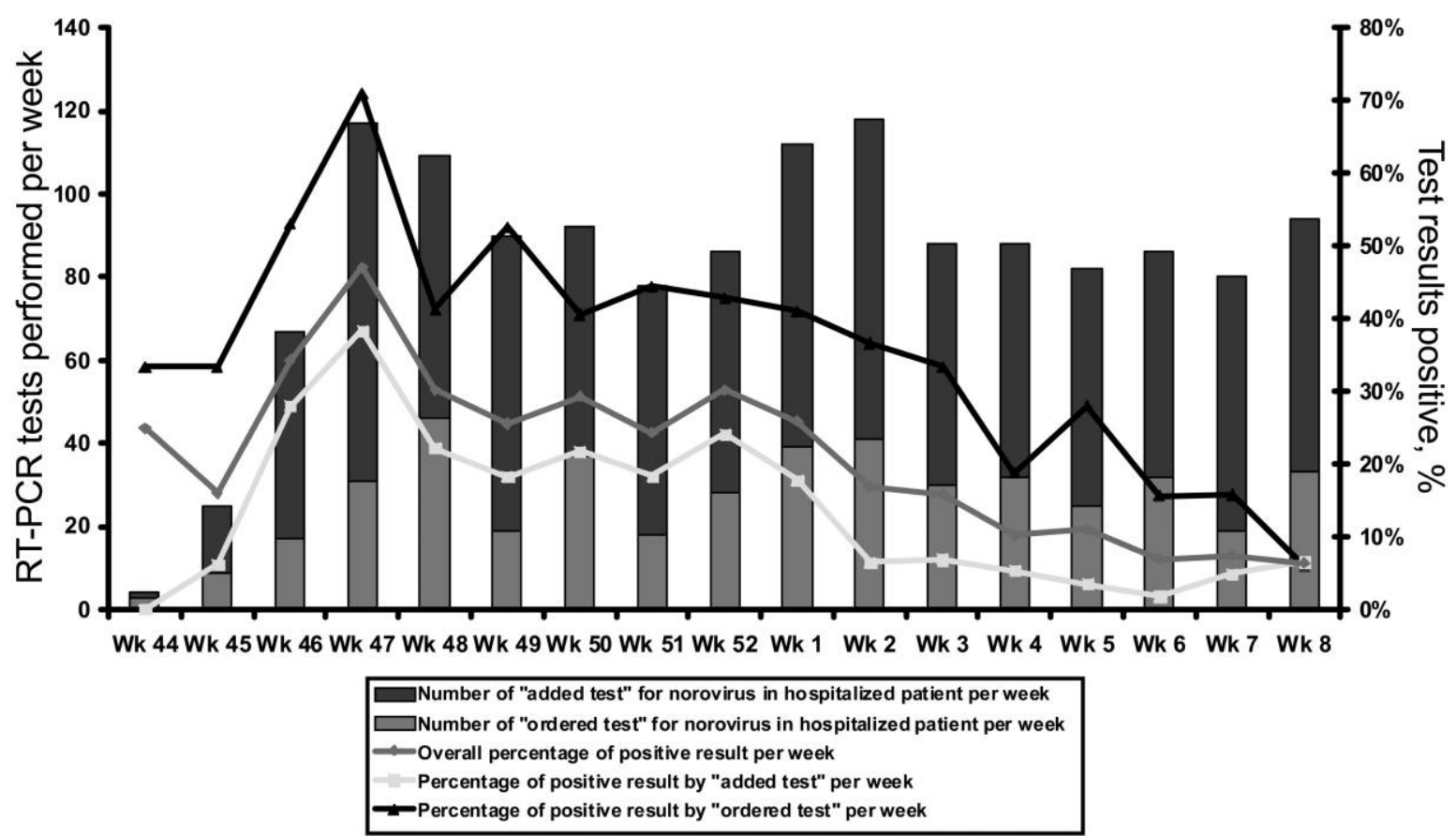

FIGURE 1. Weekly number of RT-PCR for norovirus and results of hospital patients in Queen Mary Hospital (from November 1, 2009, to February 28, 2010). An "added test" is defined as the test performed by the microbiology laboratory on all fecal specimens that were sent only for bacterial culture, Clostridium difficile culture or cytotoxin test, and rotavirus antigen detection without a request for norovirus investigation; an "ordered test" is defined as the test requested by the attending clinician. Wk 44, week 44 of 2009 , represents November 1-7, 2009; Wk 44 of 2009 to Wk 7 of 2010 is defined as a 7-day interval; Wk 8, week 8 of 2010, represents February 21-28, 2010. 


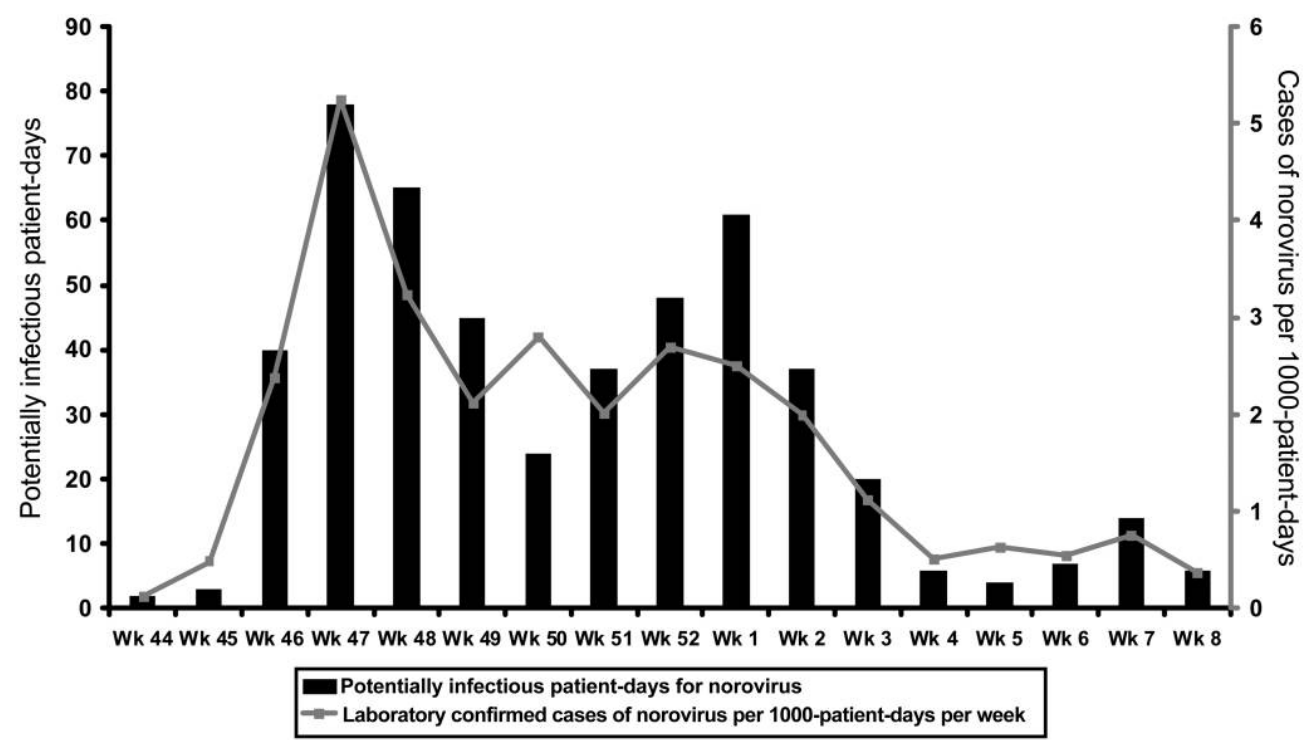

FIGURE 2. Laboratory-confirmed cases of norovirus infection per 1,000 patient-days in Queen Mary Hospital (from November 1, 2009, to February 28, 2010). Wk 44, week 44 of 2009, represents November 1-7, 2009; Wk 44 of 2009 to Wk 7 of 2010 is defined as a 7-day interval; Wk 8, week 8 of 2010, represents February 21-28, 2010.

\section{Genotyping PCR and Sequencing}

The norovirus genotyping I and II were performed using the primary and secondary seminested PCR assays and conditions described previously. ${ }^{14,15}$ All positive PCR products from either primary or secondary seminested PCR assays were purified with a QIAquick PCR purification kit (Qiagen, Hilden, Germany) and were sequenced in both directions with BigDye Teminator Cycle Sequencing Ready Reaction kit version 3.1 (Applied Biosystems, California, USA) with corresponding specific primers and an ABI 3130 Genetic Analyzer (Applied Biosystems, New Jersey, USA).

\section{Statistical Analysis}

The Fisher exact test was used to compare independent categorical variables between groups. A $P$ value of less than .05 was considered statistically significant. Computation was performed using EpiInfo 3.5.1 (CDC).

\section{RES ULTS}

\section{Infection Control Measures for Norovirus}

Between November 1, 2009, and February 28, 2010, there were 9 sessions of a staff forum held in hospital network A, with an overall attendance of 1,031 (26\%) of 3,925 healthcare workers, to update medical knowledge and infection control practice to prevent nosocomial transmission of norovirus. Including the basic and special infection control training sessions held previously, a total of 3,594 professional staff members $(91.6 \%)$ have attended our infection control training in the past 18 months.

A previous audit of hand hygiene practice showed a gradual increase in compliance in all clinical departments from $25 \%$ to $59 \%$ of opportunities between 2006 and 2008. When a yearly audit was performed in November 2009, the overall rate of hand hygiene compliance of hospital staff was consistently between $60 \%$ and $70 \%$. The consumption of alcoholbased hand rub increased from $1.5 \mathrm{~L}$ per 1,000 patient-days in $2006,18.8 \mathrm{~L}$ per 1,000 patient-days in 2007 , and $25.3 \mathrm{~L}$ per 1,000 patient-days in 2008 to $26.9 \mathrm{~L}$ per 1,000 patientdays in 2009.

\section{Epidemiology of Norovirus in Queen Mary Hospital}

During the study period, 1,612 fecal specimens from 1,153 hospitalized patients from hospital network A were received for microbiology investigation, of which 1,416 fecal specimens from 988 patients were collected in Queen Mary Hospital. Of the 1,416 fecal specimens collected from 659 patients, 957 (68\%) were classified as "added test." Two hundred fortytwo $(25 \%)$ of 988 patients tested positive for norovirus by RT-PCR; 114 (47\%) of those 242 patients had norovirus detected by our added test. Among these 114 patients, 1 patient was coinfected with Aeromonas caviae, 1 patient with toxigenic $C$. difficile, 4 patients with nontoxigenic $C$. difficile, and 1 pediatric patient with rotavirus. The weekly number of ordered tests and added tests for norovirus and the percentage of tests with a positive result are illustrated in Figure 1.

Among the 242 patients with laboratory-confirmed norovirus infection, there were 142 females and 100 males, with a median age of 57 years (range, 1 month to 101 years). Seventy-four $(31 \%)$ of 242 patients were aged 3 years or younger while $106(44 \%)$ of them were aged 70 years or older. Fifty-two (21\%) patients were long-term-care home residents. 


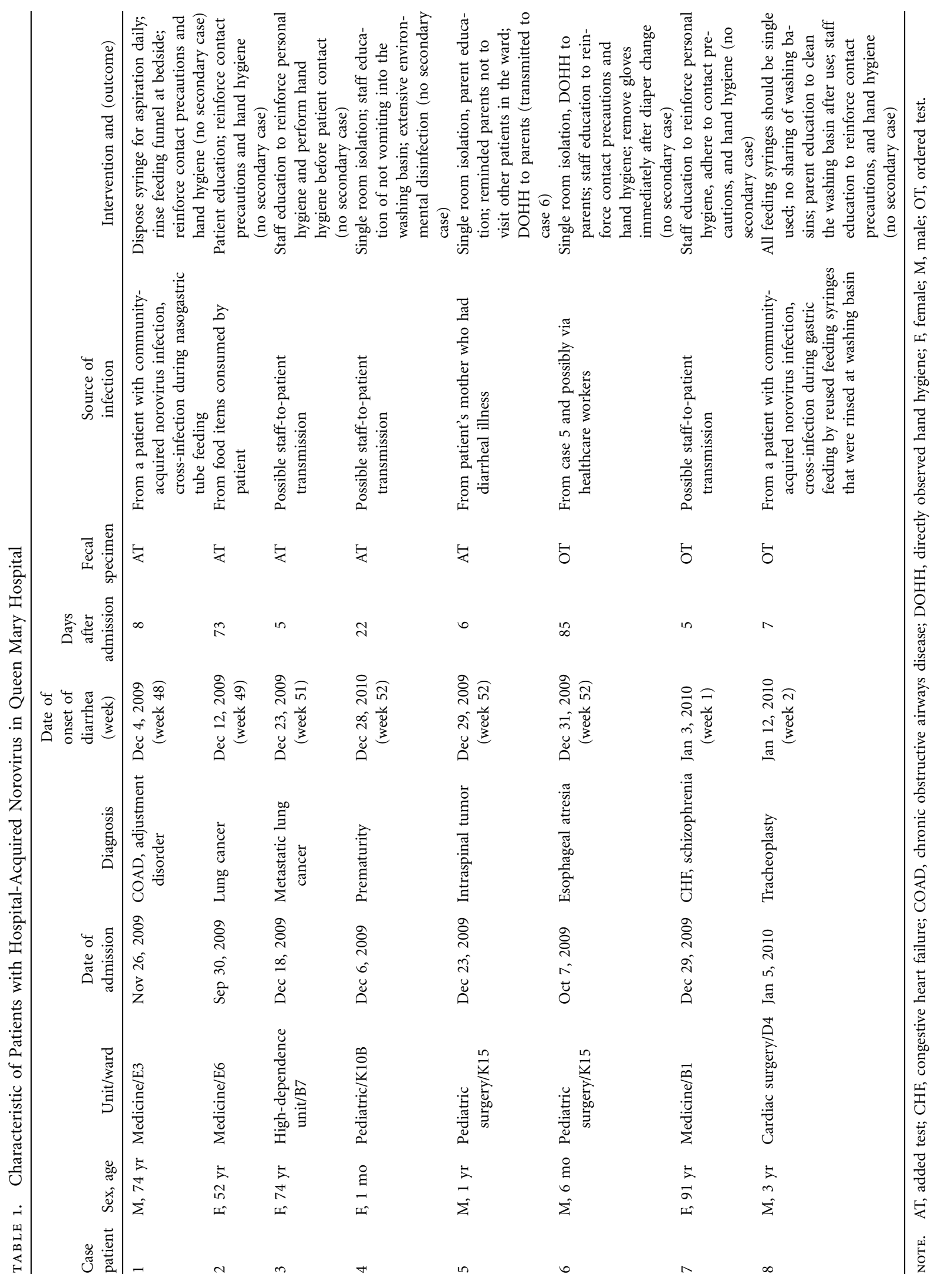


ta

\begin{tabular}{|c|c|c|c|c|c|c|c|c|}
\hline $\begin{array}{l}\text { Outbreak } \\
\text { number }\end{array}$ & $\begin{array}{c}\text { Hospital, } \\
\text { network }\end{array}$ & Clinical unit & Date of outbreak & $\begin{array}{l}\text { Duration of } \\
\text { outbreak, } \\
\text { days }\end{array}$ & $\begin{array}{l}\text { No. of infected } \\
\text { patients/no. of } \\
\text { exposed patients }\end{array}$ & $\begin{array}{c}\text { Clinical } \\
\text { attack } \\
\text { rate } \\
\text { among } \\
\text { patients, \% }\end{array}$ & $\begin{array}{l}\text { No. of infected } \\
\text { staff/no. of } \\
\text { exposed staff }\end{array}$ & $\begin{array}{c}\text { Clinical } \\
\text { attack } \\
\text { rate } \\
\text { among } \\
\text { staff, \% }\end{array}$ \\
\hline 1 & $\mathrm{~A}, \mathrm{~B}$ & Rehabilitation & Oct 29-Nov 12, 2009 & 15 & $7 / 42$ & 17 & $1 / 30$ & 3 \\
\hline 2 & $\mathrm{~B}, \mathrm{C}$ & Pediatric & Nov 14-27, 2009 & 14 & $4 / 22$ & 18 & $4 / 49$ & 8 \\
\hline 3 & C, D & Rehabilitation & Nov 19-Dec 3, 2009 & 14 & $10 / 24$ & 42 & $3 / 30$ & 10 \\
\hline 4 & $\mathrm{D}, \mathrm{E}$ & Neurosurgery & Nov 30-Dec 11, 2009 & 12 & $6 / 29$ & 21 & $8 / 36$ & 22 \\
\hline 5 & $\mathrm{E}, \mathrm{F}$ & Orthopedic & Dec 3-14, 2009 & 12 & $10 / 36$ & 28 & $0 / 41$ & 0 \\
\hline 6 & F, B & Rehabilitation & Dec 4-15, 2009 & 12 & $9 / 52$ & 17 & $1 / 28$ & 4 \\
\hline 7 & $\mathrm{G}, \mathrm{F}$ & Psychiatry & Dec 20-31, 2009 & 12 & $12 / 31$ & 39 & $0 / 31$ & 0 \\
\hline 8 & $\mathrm{H}, \mathrm{D}$ & General medicine & Dec 21-24, 2009 & 4 & $10 / 46$ & 21 & $0 / 58$ & 0 \\
\hline 9 & $\mathrm{I}, \mathrm{G}$ & General medicine & $\begin{array}{l}\text { Dec 26, 2009-Jan 2, } \\
\quad 2010\end{array}$ & 8 & $6 / 40$ & 15 & $0 / 40$ & 0 \\
\hline 10 & $\mathrm{~J}, \mathrm{G}$ & $\begin{array}{l}\text { Tuberculosis and } \\
\text { chest }\end{array}$ & Jan 5-15, 2010 & 11 & $8 / 31$ & 26 & $3 / 31$ & 10 \\
\hline 11 & $\mathrm{E}, \mathrm{F}$ & Rehabilitation & Jan 9-20, 2010 & 12 & $13 / 39$ & 33 & $1 / 31$ & 3 \\
\hline 12 & $\mathrm{H}, \mathrm{D}$ & Integrated ward & Jan $12-18,2010$ & 7 & $3 / 16$ & 19 & $0 / 45$ & 0 \\
\hline 13 & $\mathrm{E}, \mathrm{F}$ & Rehabilitation & Jan 18-Feb 4, 2010 & 18 & $15 / 36$ & 42 & $0 / 28$ & 0 \\
\hline Total & & & & & $113 / 444$ & 25 & $21 / 478$ & 4 \\
\hline
\end{tabular}

Patients were admitted into general medical wards (111 $[46 \%])$, pediatric wards (76 [31\%]), surgical wards $(21[9 \%])$, emergency admission wards $(21[9 \%])$, high dependence unit (7 $[3 \%])$, orthopedic wards $(3[1 \%])$, and obstetric wards $(3$ [1\%]). The epidemic of norovirus peaked during week 47 of 2009, when the incidence density reached 5.25 cases per 1,000 patient-days with 78 potentially infectious patient-days (Figure 2). Our infection control nurses assessed all 242 patients with norovirus infection admitted to 37 different wards at the bedside and confirmed that all patients were having symptomatic diarrhea when fecal specimens were collected for examination. Seventy-four patients had been cared for in isolation facilities upon clinical assessment, while 168 patients were managed in general wards, accounting for 496 potentially infectious patient-days with a mean duration of $3.1 \pm$ 1.1 days. Most of the patients (234 [96.7\%]) had communityacquired infection; $8(3.3 \%)$ had hospital-acquired infection (Table 1). Five (63\%) of 8 patients had infection diagnosed by the added test. Eight episodes of hospital-acquired infection in 496 potentially infectious patient-days were documented. Using the data from the corresponding period (November-February) of the preceding 12 months as historical control, there were 11 episodes of hospital-acquired infection among 24 nonisolated patients in 84 potentially infectious patient-days (mean number of potentially infectious patientdays, $3.8 \pm 3.7$ days). The incidence of hospital-acquired infection decreased from 131 to 16 cases per 1,000 potentially infectious patient-days $(P<.001)$. Assuming that added tests were not provided to hospital network A patients during this period, there were only 3 hospital-acquired norovirus infections with 173 potentially infectious patient-days. Even then, the incidence of hospital-acquired infection (15 cases per
1,000 potentially infectious patient-days) was still significantly lower than that of the historical control period (131 cases per 1,000 potentially infectious patient-days $)(P<.001)$. No nosocomial outbreak was reported during the study period.

\section{Nosocomial Outbreak of Norovirus in Hong Kong}

If we exclude hospital network $A$, where the strategic infection control measures against norovirus infection prevented any nosocomial outbreak of norovirus infection, there were 13 episodes of nosocomial outbreak of norovirus infection reported in the other 6 hospital networks $(B-G)$ in the winter of year 2009, which could be served as a concurrent control (Table 2). Ten (24\%) out of 41 public hospitals in Hong Kong were affected, involving acute medical, surgical, pediatric, orthopedic, psychiatric, and chronic rehabilitation units. In hospital E, 3 repeated outbreaks were observed. A total of 113 patients and 21 hospital staff members were infected, with the clinical attack rates ranging from $15 \%$ to $42 \%$ and from $3 \%$ to $22 \%$, respectively. No visitors were involved. The median duration of the nosocomial outbreak was 12 days (418 days). Nine (69\%) of 13 nosocomial outbreaks were reported in November and December 2009. The overall incidence of hospital-acquired norovirus infection was 0.06 cases per 1,000 patient-days among 6 hospital networks (B-G), which was significantly higher than that of hospital network A ( 0.03 cases per 1,000 patient-days $)(P=.015)$.

\section{Molecular Diagnosis and Phylogenetic Analysis of Norovirus}

Forty-six (19\%) out of 242 strains of norovirus isolated during the study period were selected for sequencing (Figure 

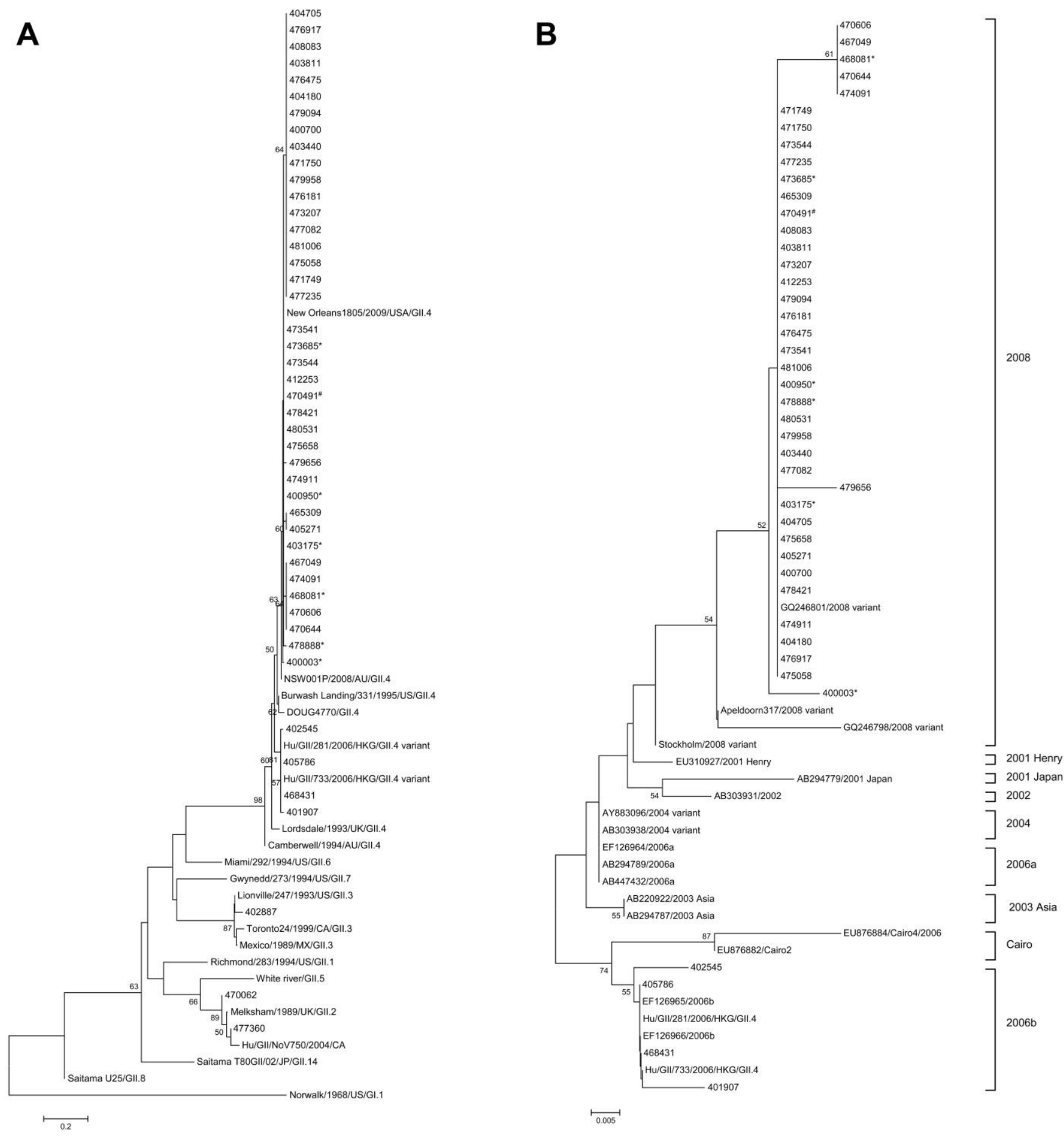

FIGURE 3. Phylogenetic dendrogram of norovirus. A, Phylogenetic dendrogram generated based on the ORF $1 / 2$ junction region of norovirus using novel sequences in isolates from this study $(n=46)$ and GenBank $(n=20)$; Norowalk/1968/US was used as the outgroup. B, A further subtree generated from the genotype group II.4 variants $(n=43)$ and GenBank $(n=20)$. Numbers on the branches represent bootstrap values after 1,000 replications, and bootstrap values $\geq 50$ are shown. An asterisk represents the strains collected from patients with hospital-acquired infection. A number sign represents the 470491 strain collected from index patients as a source of nosocomial patient-to-patient transmission. 
$3 A)$. Except for 3 patients with community-acquired norovirus due to genogroup II.2, genogroup II.3, all other patients' isolates belonged to genogroup II. 4 variant, of which 4 isolates were closed to genogroup II.4 variant 2006 b and 39 isolates, including 6 isolates from patients with nosocomial onset of diarrhea, were closed to the 2008 variant $^{16}$ (Figure $3 B$ ).

\section{I S C USS I O N}

Norovirus has caused numerous outbreaks, affecting about 25\% of all public hospitals in Hong Kong during the winter months of 2009, involving all clinical disciplines from acute to long-term patient care areas. With the implementation of strategic infection control measures in Queen Mary Hospital and its 4 network hospitals in 2009, we were able to prevent any nosocomial outbreak of norovirus infection and reduce the incidence of hospital-acquired norovirus infection from 131 to 16 cases per 1,000 potentially infectious patient-days. The concept of using an added test for early recognition of any index case was simple but innovative. Without affecting the clinical workflow of the frontline medical and nursing staff, all fecal specimens referred to the microbiology laboratory were tested for norovirus RT-PCR, regardless of the clinician's request. The provision of rapid laboratory diagnostic testing facilitated timely intervention by our infection control team, who provided advice as to patient placement, isolation precautions, and thorough environmental disinfection with sodium hypochlorite $(1,000 \mathrm{ppm})$, thus preventing an outbreak.

The overall compliance with standard infection control practice was satisfactory among our frontline healthcare workers, especially after the intensive training in infection control during the emergence of pandemic influenza in mid2009 in Hong Kong. ${ }^{9}$ The rate of compliance with hand hygiene practice approached $70 \%$ in November 2009 among all ranks of healthcare workers, and the consumption of alcoholbased hand rub solution increased even before our implementation of enhanced infection control measures. In this connection, none of the healthcare workers reported diarrhea after caring for the infected patients upon contact tracing. In addition, directly observed hand hygiene (DOHH) practice, which has proved effective in outbreak prevention and control, ${ }^{17,18}$ was further enforced by our infection control nurses when there was any evidence of nosocomial acquisition of norovirus. This might be useful in preventing outbreaks evolving from episodic cases. However, the use of alcoholbased hand rub has been questioned in the control and prevention of norovirus, a nonenveloped RNA virus, especially if the concentration of ethanol is less than $80 \%$ and the contact time is less than 20 seconds. ${ }^{19,20}$ Although the WHO formulation of alcohol-based hand rub, formula I containing ethanol $(80 \% \mathrm{vol} / \mathrm{vol})$, has been used in our center, further investigation is needed to examine the effectiveness of alcohol-based hand rub against norovirus infection.

Molecular sequencing of norovirus isolated during the win- ter of 2009 demonstrated a predominant strain of genotype II.4 variant. This epidemic strain has emerged in the United States and European countries since 2002 and arrived in Hong Kong in 2006, resulting in an increased number of nosocomial outbreaks. ${ }^{2,4,21}$ It is the first demonstration that nosocomial transmission of this epidemic strain of norovirus can be prevented by the implementation of strategic infection control measures.

Cost-effective analysis based on previous studies in Hong Kong showed that the minimum cost of each nosocomial outbreak of norovirus in Hong Kong is around HK\$311,580 (US\$40,000). ${ }^{4}$ The minimum cost of 13 reported nosocomial outbreaks in Hong Kong is HK\$4,050,540 (US\$520,000). Since the extra cost for the strategic infection control measures is the added test for rapid molecular diagnostic of norovirus, HK\$200 (US\$25) per test, the minimum cost for added tests during the winter in Hong Kong is $\mathrm{HK} \$ 1,400,000$ (US\$180,000) (approximately 1,000 added tests per hospital network times 7 networks in Hong Kong times HK\$200). Performing these added tests would result in a potential savings of around HK\$2,600,000 (US\$340,000).

There are several limitations in this study. First, the number of hospital-acquired norovirus infections in our cohort was low, and therefore the risk factors for nosocomial transmission of norovirus cannot be investigated from a statistical perspective. However, the risk factors of nosocomial acquisition of norovirus have been well reported; lapses in infection control practice in nonisolation facilities and introduction of infection by a contaminated food source or symptomatic healthcare workers were found to be important risk factors. $^{22-27}$ In fact, all of our hospital-acquired norovirus infections occurred because of one of the reasons mentioned above. Second, the incidence of hospital-acquired norovirus infection in the study and historical control periods should be carefully interpreted because of the difference in case finding which might potentially introduce bias in the concurrent comparison of hospital-acquired norovirus infection between different hospital networks. The total number of nosocomial norovirus outbreaks in Hong Kong during the study period might be underestimated because private hospitals are not required to notify the Hospital Authority of an outbreak. However, the impact is probably trivial because the public hospital system serves more than $90 \%$ of Hong Kong's population. Frequent reports of norovirus outbreaks in public hospitals involving a wide range of clinical specialties indicated that the promotion of our strategic infection control measures is justified to decrease nosocomial transmission of norovirus.

\section{ACKNOWLEDGMENTS}

We thank Dr P. Y. Leung of the Hospital Authority for facilitating this study. This work was partly funded by Suen Chi Sun Charitable Foundation and Research Fund for the Control of Infectious Diseases (RFCID). 
Potential conflicts of interest. All authors report no conflicts of interest relevant to this article.

Address reprint requests to K. Y. Yuen, Carol Yu Centre for Infection and Division of Infectious Diseases, Department of Microbiology, University of Hong Kong, Queen Mary Hospital, Pokfulam Road, Pokfulam, Hong Kong Special Administrative Region, China (kyyuen@hkucc.hku.hk).

\section{REFERENCES}

$\rightarrow$ 1. Kampf G, Kramer A. Epidemiologic background of hand hygiene and evaluation of the most important agents for scrubs and rubs. Clin Microbiol Rev 2004;17:863-893.

$\rightarrow$ 2. Lopman B, Vennema H, Kohli E, et al. Increase in viral gastroenteritis outbreaks in Europe and epidemic spread of new norovirus variant. Lancet 2004;363:682-688.

$\rightarrow$ 3. Ho EC, Cheng PK, Lau AW, Wong AH, Lim WW. Atypical norovirus epidemic in Hong Kong during summer of 2006 caused by a new genogroup II/4 variant. J Clin Microbiol 2007; 45:2205-2211.

$\rightarrow$ 4. Tsang OT, Wong AT, Chow CB, Yung RW, Lim WW, Liu SH. Clinical characteristics of nosocomial norovirus outbreaks in Hong Kong. J Hosp Infect 2008;69:135-140.

$\rightarrow$ 5. Hansen S, Stamm-Balderjahn S, Zuschneid I, et al. Closure of medical departments during nosocomial outbreaks: data from a systematic analysis of the literature. J Hosp Infect 2007;65:348353.

$\rightarrow$ 6. Wu HM, Fornek M, Schwab KJ, et al. A norovirus outbreak at a long-term-care facility: the role of environmental surface contamination. Infect Control Hosp Epidemiol 2005;26:802-810.

$\rightarrow$ 7. Johnston CP, Qiu H, Ticehurst JR, et al. Outbreak management and implications of a nosocomial norovirus outbreak. Clin Infect Dis 2007;45:534-540.

$\rightarrow$ 8. Cheng VC, Tai JW, Wong LM, et al. Prevention of nosocomial transmission of swine-origin pandemic influenza virus $\mathrm{A} / \mathrm{H} 1 \mathrm{~N} 1$ by infection control bundle. J Hosp Infect 2010;74:271-277.

9. Hong Kong Observatory. 2010. Climate of Hong Kong. http:// www.hko.gov.hk/cis/climahk_e.htm Accessed July 22, 2010.

$\rightarrow$ 10. Billgren M, Christenson B, Hedlund KO, Vinjé J. Epidemiology of Norwalk-like human caliciviruses in hospital outbreaks of acute gastroenteritis in the Stockholm area in 1996. J Infect 2002; 44:26-32.

11. Tai JW, Mok ES, Ching PT, Seto WH, Pittet D. Nurses and physicians' perceptions of the importance and impact of healthcare-associated infections and hand hygiene: a multi-center exploratory study in Hong Kong. Infection 2009;37:320-333.

$\rightarrow$ 12. Pittet D, Allegranzi B, Boyce J; World Health Organization World Alliance for Patient Safety First Global Patient Safety Challenge Core Group of Experts. The World Health Organization guidelines on hand hygiene in health care and their consensus recommendations. Infect Control Hosp Epidemiol 2009; 30:611-622.

$\rightarrow$ 13. Chan KH, Yam WC, Pang CM, et al. Comparison of the NucliSens easyMAG and Qiagen BioRobot 9604 nucleic acid extraction systems for detection of RNA and DNA respiratory viruses in nasopharyngeal aspirate samples. J Clin Microbiol 2008;46:2195-2199.

14. Kageyama T, Kojima S, Shinohara M, et al. Broadly reactive and highly sensitive assay for Norwalk-like viruses based on realtime quantitative reverse transcription-PCR. J Clin Microbiol 2003;41:1548-1557.

15. Gallimore CI, Iturriza-Gomara M, Xerry J, Adigwe J, Gray JJ. Inter-seasonal diversity of norovirus genotypes: emergence and selection of virus variants. Arch Virol 2007;152:1295-1303.

16. Belliot G, Kamel AH, Estienney M, Ambert-Balay K, Pothier P. Evidence of emergence of new GGII.4 norovirus variants from gastroenteritis outbreak survey in France during the 2007-to2008 and 2008-to-2009 winter seasons. J Clin Microbiol 2010; 48:994-998.

17. Cheng VC, $\mathrm{Wu} \mathrm{AK}$, Cheung $\mathrm{CH}$, et al. Outbreak of human metapneumovirus infection in psychiatric inpatients: implications for directly observed use of alcohol hand rub in prevention of nosocomial outbreaks. J Hosp Infect 2007;67:336-343.

$\rightarrow$ 18. Cheng VC, Tai JW, Ho YY, Chan JF. Successful control of norovirus outbreak in an infirmary with the use of alcohol-based hand rub. J Hosp Infect 2009;72:370-371.

$\rightarrow$ 19. Doultree JC, Druce JD, Birch CJ, Bowden DS, Marshall JA. Inactivation of feline calicivirus, a Norwalk virus surrogate. $J$ Hosp Infect 1999;41:51-57.

20. Gehrke C, Steinmann J, Goroncy-Bermes P. Inactivation of feline calicivirus, a surrogate of norovirus (formerly Norwalk-like viruses), by different types of alcohol in vitro and in vivo. $J$ Hosp Infect 2004;56:49-55.

21. Siebenga JJ, Vennema H, Zheng DP, et al. Norovirus illness is a global problem: emergence and spread of norovirus GII.4 variants, 2001-2007. J Infect Dis 2009;200:802-812.

22. Lopman BA, Andrews N, Sarangi J, Vipond IB, Brown DW, Reacher MH. Institutional risk factors for outbreaks of nosocomial gastroenteritis: survival analysis of a cohort of hospital units in south-west England, 2002-2003. J Hosp Infect 2005;60: 135-143.

23. Cáceres VM, Kim DK, Bresee JS, et al. A viral gastroenteritis outbreak associated with person-to-person spread among hospital staff. Infect Control Hosp Epidemiol 1998;19:162-167.

24. Marx A, Shay DK, Noel JS, et al. An outbreak of acute gastroenteritis in a geriatric long-term-care facility: combined application of epidemiological and molecular diagnostic methods. Infect Control Hosp Epidemiol 1999;20:306-311.

25. Weber DJ, Sickbert-Bennett EE, Vinjé J, et al. Lessons learned from a norovirus outbreak in a locked pediatric inpatient psychiatric unit. Infect Control Hosp Epidemiol 2005;26:841-843.

26. Iijima Y, Tanaka S, Ohishi H. Multiple outbreaks of gastroenteritis due to a single strain of genotype GII/4 norovirus in Kobe, Japan, 2006: risk factors for norovirus spread in health care settings. Jpn J Infect Dis 2008;61:419-422.

27. Turcios-Ruiz RM, Axelrod P, St John K, et al. Outbreak of necrotizing enterocolitis caused by norovirus in a neonatal intensive care unit. J Pediatr 2008;153:339-344. 